\title{
LINEAR EXTENSIONS AND LINEAR LIFTINGS IN SUBSPACES OF $C(X)$
}

\author{
EGGERT BRIEM
}

\begin{abstract}
If $X$ is a compact Hausdorff space, if $B$ is a closed subspace of $C(X)$ and if $F$ is a closed subset of $X$, conditions are given which ensure the existence of a linear extension operator of norm 1 from the restriction space $\left.B\right|_{F}$ to $B$.
\end{abstract}

1. In this note we study the following two problems. Given a compact Hausdorff space $X$ and a uniformly closed subspace $B$ of $C(X)$, the space of all continuous complex-valued functions on $X$, which separates points of $X$ and contains the constant functions.

I. If $F$ is a closed subset of $X$ with certain properties defined below ( $F$ is an $M$-set), when is there a linear extension operator of norm 1 from the restriction space $\left.B\right|_{F}$ into $B$ ?

II. If $J$ is a subspace of $B$ which is an $M$-ideal, does there exist a continuous linear inverse to the canonical map of $B$ into $B / J$ ?

$M$-sets which are considered in the first problem are the complex-space analogues of split faces of compact convex sets and they are also the function space analogues of the generalized peak sets for function algebras. An $M$-ideal in $B$ is just the space of functions which vanish on some $M$-set for $B$ so that the second problem is another formulation of the first once it has been seen that $\left.B\right|_{F}$ is isometrically isomorphic to $B / J$.

In this note we show that for an $M$-set $F$ problem I has a solution if $\left.B\right|_{F}$ has the metric approximation property. We then use a construction of Davie [8] to show that if $F$ is metric and if problem I has a solution in general then $\left.B\right|_{F}$ has the metric approximation property.

The idea in the proof of the existence of the linear extension operator is to show that one can construct finite dimensional extensions of the same kind as the ones constructed in the papers of Michael and Pełczyński [10] and Davie [8]. The construction of the finite dimensional extensions involves among others ideas from a paper of Rao [11] and the construction is actually reduced to showing the existence of an extension of a single function on a new $M$-set in a space larger than $X$, the proof of the existence of this extension is then a slight modification of the proofs in the papers of Alfsen and Hirsberg [3] and the author and Rao [7].

In the case when $\left.B\right|_{F}$ is a $\pi_{1}$-space, solutions to problems I and II can be

Received by the editors June 19, 1974 .

AMS (MOS) subject classifications (1970). Primary 46E15; Secondary 46A30.

Key words and phrases. Linear extension, $M$-set, $M$-ideal, linear inverse, metric approximation property.

(1) American Mathematical Society 1976 
obtained from Ando's paper [5] and in the case when $B$ is a real space the problems have been solved by T. B. Andersen [4].

We note that Theorem 7 of this note is more general than corresponding theorems in [8] and [10] since the property that $F$ be an $M$-set is weaker than the property that $\left(\left.B\right|_{F}, B\right)$ have the bounded extension property (as can be seen e.g. when $f$ is a split face of a compact convex set $X$ ).

2. If $J$ is a closed subspace of a Banach space $B$ then $J$ is said to be an $M$ ideal if its annihilator $J^{\perp}$ in $B^{*}$ is an $L$-ideal which means that $J^{\perp}$ is the range of a projection $e$ such that

$$
\|p\|=\|e(p)\|+\|(1-e)(p)\| \text { for all } p \in B^{*} .
$$

In this note $B$ is a closed subspace of $C(X)$, the space of all continuous complex-valued functions on a compact Hausdorff space $X$ equipped with the supremum norm. We assume that $B$ separates points of $X$ and contains the constant functions. The state space of $B$ is the set $S_{B}=\left\{p \in B^{*}: p(1)=\|p\|\right.$ $=1$ \}. When $S_{B}$ is equipped with the $w^{*}$-topology it is a $w^{*}$-compact convex subset of $B^{*}$ and the natural map of $X$ into $S_{B}$ is a homeomorphism so that we can consider $X$ as a subset of $S_{B}$. The set of extreme points for $S_{B}$ is a subset of $X$, denoted by $\partial_{B} X$ and called the Choquet boundary of $B$. We let $M(X)$ denote the set of all regular complex Borel measures on $X$ and $B^{\perp}$ those measures in $M(X)$ which annihilate $B$. Further, we let $M\left(\partial_{B} X\right)$ denote the set of those $\mu \in M(X)$ for which the total variation measure $|\mu|$ is maximal in Choquet's ordering of positive measures on $S_{B}$.

A closed subset $F$ of $X$ with the following three properties is called an $M$-set.

(i) $F$ is $B$-convex, i.e.,

$$
F=\left\{x \in X:|b(x)| \leqslant\|b\|_{F}\right\}
$$

where $\|b\|_{F}=\sup \{|b(y)|: y \in F\}$ for $b \in B$,

(ii) if $\mu \in B^{\perp} \cap M\left(\partial_{B} X\right)$ then $\left.\mu\right|_{F} \in B^{\perp}$,

(iii) if $\mu$ and $\nu$ are two probability measures such that $\mu-\nu \in B^{\perp}, \operatorname{supp}(\mu)$ $\subseteq F$ and $\nu \in M\left(\partial_{B} X\right)$ then also supp $(\nu) \subseteq F$.

In [9] it is proved that a closed subspace $J$ of $B$ is an $M$-ideal if and only if there exists an $M$-set $F$ for $B$ such that $J=\left\{b \in B:\left.b\right|_{F} \equiv 0\right\}$.

A slight modification of the proofs in [3] or [7] shows that if $F$ is an $M$-set then each function in $\left.B\right|_{F}$ has a norm preserving extension to a function in $B$. Also, the $F$-sets are generalized peak sets (i.e. intersections of peak sets [3, Theorem 5.4 and remark]) so that the $M$-sets might alternatively be characterized as those generalized peak sets $F$ for $B$ for which $\left.\mu\right|_{F} \in B^{\perp}$ for all $\mu \in B^{\perp} \cap M\left(\partial_{B} X\right)$. We note that conditions (i) and (iii) in the definition of $M$-sets may be replaced by the condition that co $(F)$, the closed convex hull of $F$ in $S_{B}$, is a face of $S_{B}$ with $F=X \cap \overline{c o}(F)$. It is then easy to show that if $F$ is an $M$-set then co $(F)$ is a split face of $S_{B}$ [9]. If $\overline{c o}(F)^{\prime}$ denotes the complementary face of co $(F)$, i.e., the union of all faces of $S_{B}$ which are disjoint from co $(F)$ then the complementary set of the $M$-set $F$ is the set $F^{\prime}=\overline{c o}(F)^{\prime} \cap X$. The set $F^{\prime}$ is a Borel-set and if $\mu$ is a maximal measure then $|\mu|\left(X \backslash\left(F \cup F^{\prime}\right)\right)=0 \quad[1$, Chapter II $]$.

It is sometimes useful to have a real isomorphic representation of $B$ as the 
space of all real-valued continuous affine functions on a certain compact convex set $K$. In [6] it is proved that if $Z$ is the $w^{*}$-compact convex subset $B^{*}$ given by $Z=\operatorname{co}\left(S_{B} \cup-i S_{B}\right)$ then the embedding $b \rightarrow \theta b$, where $\theta b(z)$ $=\operatorname{Re} b(z)$ for $z \in Z$, is a bicontinuous real-linear isomorphism of $B$ onto $A(Z)$, the Banach space of all real-valued continuous affine functions on $Z$. The $M$-sets can then be characterized as follows [9]: A closed $B$-convex subset $F$ of $X$ is an $M$-set for $B$ if and only if co $(F \cup-i F)$ is a split face of $Z$. With the aid of this characterization we prove a proposition which will be useful later.

Proposition 1. Let $F$ be an $M$-set for $B$ and let $\mu \in M\left(\partial_{B} X\right)$. Then for each $\left.b_{0} \in B\right|_{F}$ with $\left\|b_{0}\right\|_{F}<1$, for each compact subset $E$ of $F^{\prime}$ and for each $\varepsilon>0$ there is a function $b \in B$ with $\left.b\right|_{F}=b_{0},\|b\|<2$ and $\|b\|_{E}<\varepsilon$ (here $\|b\|_{E}$ $=\sup \{|b(y)|: y \in E\})$.

Proof. Suppose first that $\operatorname{Re} b_{0}>0$ and that $\operatorname{Im} b_{0}>0$. The set

$$
\overline{\mathrm{co}}(F \cup-i F)
$$

is a split face of $Z=\operatorname{co}\left(S_{B} \cup-i S_{B}\right)$ and the complementary face of $\overline{c o}(F \cup-i F)$ is easily seen to contain $E \cup-i E$. By [1, Theorem II.6.18] there is a net $\left\{b_{\alpha}\right\}$ in $B$ such that $\operatorname{Re} b_{\alpha}$ decreases to $\operatorname{Re} b_{0}$ on $F$ and to zero on $E$ and such that $\operatorname{Im} b_{\alpha}$ decreases to $\operatorname{Im} b_{0}$ on $F$ and to 0 on $E$, and we may assume $\left\|b_{\alpha}\right\|<2$. This shows the existence of a function $b_{1} \in B$ such that $\left\|b_{1}-b_{0}\right\|_{F}<\varepsilon$, such that $\left\|b_{1}\right\|_{E}<\varepsilon$ and such that $\left\|b_{1}\right\|<2-\left\|b_{1}-b_{0}\right\|_{F}$. If $b_{2}$ is a normpreserving extension of $\left.b_{1}\right|_{F}-b_{0}$ to a function in $B$, then $b=b_{1}-b_{2}$ is the required function. The result is then seen to hold for all constant functions and then for all functions $\left.b_{0} \in B\right|_{F}$ whether or not both $\operatorname{Re} b_{0}$ and $\operatorname{Im} b_{0}$ are positive.

We now begin to look for the linear extension operator and begin with the finite dimensional case.

THEOREM 2. Let $F$ be an $M$-set for $B$ and let $L$ be a finite dimensional subspace of $\left.B\right|_{F}$. Then there exists a linear operator $u$ of norm 1 from $L$ into $B$ such that $\left.u\left(b_{0}\right)\right|_{F}=b_{0}$ for all $\left.b_{0} \in B\right|_{F}$.

The proof of Theorem 2 will be reduced to the proof of the existence of normpreserving extensions from $M$-sets.

Let $\left\{b_{1}, \ldots, b_{n}\right\}$ be a basis for $L$ and let

$$
K=\left\{z=\left(z_{1}, \ldots, z_{n}\right) \in C^{n}:\left\|\sum_{i=1}^{n} z_{i} b_{i}\right\|_{F} \leqslant 1\right\} .
$$

Then $K$ is a compact convex subset of $C^{n}$ containing 0 . We define two subspaces $A$ and $A_{0}$ of $C(K \times X)$, the space of all continuous complex valued functions on $K \times X$ :

$$
\begin{aligned}
& A=\left\{f \in C(K \times X): \exists c_{0}, \ldots, c_{n} \in B\right. \text { such that } \\
& \left.\qquad f(z, x)=\sum_{i=1}^{n} z_{i} c_{i}(x)+c_{0}(x) \text { for }(z, x) \in K \times X\right\}
\end{aligned}
$$


and

$$
\begin{aligned}
& A_{0}=\left\{g \in C(K \times X): \exists c_{1}, \ldots, c_{n} \in B\right. \text { such that } \\
& \left.\qquad g(z, x)=\sum_{i=1}^{n} z_{i} c_{i}(x) \text { for }(z, x) \in K \times X\right\} .
\end{aligned}
$$

The spaces $A_{0}$ and $A$ are uniformly closed subspaces of $C(K \times X), A_{0}$ is a subspace of $A$, and $A$ separates the points of $K \times X$ and contains the constant functions. The idea is now to show that the function $\left.h_{0} \in A_{0}\right|_{K \times F}$ given by $h_{0}(z, x)=\sum_{i=1}^{n} z_{i} b_{i}(x)$ for $(z, x) \in K \times F$ has a normpreserving extension to a function $h \in A_{0}$. Once this has been established the existence of the desired linear extension from $L$ into $B$ is an easy consequence.

Lemma 3. Let $\partial_{A}$ denote the Choquet boundary for $A$ and let $\mu \in M\left(\partial_{A}\right)$. Then $\mu$ has no mass outside $(K \times F) \cup\left(K \times F^{\prime}\right)$.

Proof. Let $1_{K \times F}$ denote the characteristic function of $K \times F$ and let $\hat{1}_{K \times F}$ be the upper envelope of $K \times F$, i.e., $\hat{\mathrm{l}}_{K \times F}(z, x)=\inf \{\operatorname{Re} f(z, x): f$ $\in A$ and $\left.\operatorname{Re} f>1_{X \times F}\right\}$. The $M$-set $F$ is a generalized peak set for $B$ which in turn implies that $K \times F$ is a generalized peak set for $A$. Then it follows from [1, Chapter II] that $\mu$ has no mass outside the set $\left\{(z, x) \in K \times X: \hat{\mathrm{l}}_{K \times F}(z, x)\right.$ $=1$ or 0$\}$. If we define $\hat{\mathrm{l}}_{F}$ relative to $B$ in a similar way as $\hat{\mathrm{l}}_{K \times F}$ was defined relative to $A$ then $\hat{\mathrm{l}}_{F}(x)=1$ only when $x \in F$ and $\hat{\mathrm{l}}_{F}(x)=0$ only when $x \in F^{\prime}$. Suppose $\hat{1}_{K \times F}\left(z^{\prime}, x^{\prime}\right)=0$; then it follows from the definition of the upper envelope that $\hat{\mathrm{l}}_{F}\left(x^{\prime}\right)=0$. Conversely, if $\hat{\mathrm{l}}_{F}\left(x^{\prime}\right)=0$ then it is clear that $\hat{\mathrm{l}}_{K \times F}\left(z, x^{\prime}\right)=0$ for all $z \in K$. Thus $\left\{(z, x) \in K \times X: \hat{\mathrm{l}}_{K \times F}(z, x)=1\right.$ or 0$\}$ $=(K \times F) \cup\left(K \times F^{\prime}\right)$ which concludes the proof of the lemma.

Proposition 4. $K \times F$ is an $M$-set for $A$ and $\left.\mu\right|_{K \times F} \in A_{0}^{\perp}$ for all $\mu$ $\in A_{0}^{\perp} \cap M\left(\partial_{A}\right)$.

Proof. Suppose $\mu \in A^{\perp} \cap M\left(\partial_{A}\right)$ and let $\left.f_{0} \in A\right|_{K \times F}$. By regularity of the measure $\mu$ we can for each $\varepsilon>0$ find a compact subset $E$ of $F^{\prime}$ such that $|\mu|(K \times X \backslash((K \times F) \cup(K \times E)))<\varepsilon$. Let $c_{0}, \ldots, c_{n} \in B$ be such that $f_{0}(z, x)=\sum_{i=1}^{n} z_{i} c_{i}(x)+c_{0}(x)$ for all $(z, x) \in K \times F$. By Proposition 1 there exist $c_{0}^{\prime}, \ldots, c_{n}^{\prime} \in B$ such that $\left.c_{i}^{\prime}\right|_{F}=\left.c_{i}\right|_{F},\left\|c_{i}^{\prime}\right\|_{E}<\varepsilon$ and $\left\|c_{i}^{\prime}\right\|<2\left\|c_{i}\right\|_{F}, i$ $=0, \ldots, n$. Then if $f(z, x)=\sum_{i=1}^{n} z_{i} c_{i}^{\prime}(x)+c_{0}^{\prime}(x)$ for all $(z, x) \in K \times X$ we have

$$
\left|\int_{K \times F} f_{0} d \mu\right|=\left|\int_{K \times F^{\prime}} f d \mu\right| \leqslant \varepsilon\left[2(n+1) C\left\|f_{0}\right\|_{K \times F}+C^{\prime}(n+1)\right]
$$

where $C$ and $C^{\prime}$ are constants depending only on the set $K$. Since $\varepsilon>0$ was arbitrary we conclude that $\left.\mu\right|_{K \times F} \in A^{\perp}$. As we had already observed $K \times F$ is a generalized peak set for $A$ and using one of the alternative characterizations of $M$-sets it follows that $K \times F$ is an $M$-set for $A$.

By taking the function $c_{0}$ in the proof above to be the zero function the same proof shows that if $\mu$ belongs to $A_{0}^{\perp} \cap M\left(\partial_{A}\right)$ then $\left.\mu\right|_{K \times F}$ also belongs to $A_{0}^{\perp}$.

We now have the following set up: There is a compact Hausdorff space $Y(=K \times X)$, a closed point-separating subspace $A$ of $C(Y)$ containing the 
constant functions, an $M$-set $G(=K \times F)$ for $A$ and a closed subspace $A_{0}$ of $A$ with the property that $\left.\mu\right|_{G} \in A_{0}^{\perp}$ for all $\mu \in A_{0}^{\perp} \cap M\left(\partial_{A} Y\right)$. The next lemma contains a slightly stronger result than needed for the proof of Theorem 2 but this stronger result is used in a later proposition.

Lemma 5. Let $\left.a_{0} \in A_{0}\right|_{G}$ with $\left\|a_{0}\right\|_{G} \leqslant 1$, let $a \in A_{0}$ be an extension of $a_{0}$ with $\|a\|<1+\alpha, \alpha$ some positive number. Then there is another extension $a^{\prime} \in A_{0}$ of $a$ such that $\left\|a^{\prime}\right\| \leqslant 1$ and such that $\left\|a-a^{\prime}\right\| \leqslant \alpha$.

Proof. We extend $a_{0}$ to a function $\bar{a}_{0}$ on $Y$ as follows: If $x \in Y$ and if $\lambda_{x}$ is a probability measure in $M\left(\partial_{A} Y\right)$ representing $x$ w.r.t. $A$ we define

$$
\bar{a}_{0}(x)=\int_{G} a_{0} d \lambda_{x} .
$$

By property (ii) in the definition of an $M$-set, $\bar{a}_{0}$ is well defined, and from property (iii) it follows that $\bar{a}_{0}(x)=a_{0}(x)$ if $x \in G$. Clearly $\bar{a}_{0}(x)=0$ if $x$ $\in \partial_{A} Y \backslash G$. The proof of [3, Lemma 3.1] shows that $\bar{a}_{0}$ is a Borel function and that

$$
\int \bar{a}_{0} d \mu=0 \text { for all } \mu \in A_{0}^{\perp} .
$$

Further, it follows from the proof of [3, Proposition 4.4] that for each $\left.c_{0} \in A_{0}\right|_{G}$ there is a function $c \in A_{0}$ extending $c_{0}$ with $\|c\| \leqslant\left\|c_{0}\right\|_{G}+\varepsilon$.

Let now $0<\beta<\alpha$. We shall show, that there is an extension $a_{1}$ $\in A_{0}$ of $a_{0}$ with $\left\|a_{1}\right\|<1+\beta$ and with $\left\|a-a_{1}\right\|<\alpha-\beta$. Once this has been proved the existence of $a^{\prime}$ is clear.

Pick $\varepsilon>0$ such that $\|a\|+\varepsilon<1+\alpha$ and such that $\varepsilon<\min (\alpha-\beta, \beta)$ and put

$$
\begin{array}{r}
H=\left\{f \in C(Y):\|f-a\|_{G}<\varepsilon,\|f\|<1+\beta-\varepsilon\right. \\
\quad \text { and }\|f-a\|<\alpha-\beta-\varepsilon\} .
\end{array}
$$

Then $H$ is a nonempty convex open subset of $C(Y)$. We claim that $H \cap A_{0} \neq \varnothing$. Suppose for contradiction that $H \cap A_{0}=\varnothing$. Then, by the Hahn-Banach and the Riesz representation theorem there is a nonzero measure $\mu \in A_{0}^{\perp}$ such that

$$
\operatorname{Re}\left(\int \phi d \mu\right)>0 \text { for all } \phi \in H .
$$

We now put $t=(1+\beta) /(1+\alpha-\varepsilon)$ and $b=\bar{a}_{0}+t\left(a-\bar{a}_{0}\right)$. If now $x \in Y$ and if $\lambda_{x}$ is any probability measure in $M\left(\partial_{A} Y\right)$ which represents $x$ w.r.t. $A$ then $\delta_{x}-\lambda_{x}$ is in $A_{0}^{\perp}\left(\delta_{x}\right.$ denotes unit mass at $\left.x\right)$. Thus by definition of $\bar{a}_{0}$ we have

$$
\bar{a}_{0}(x)=\int \bar{a}_{0} d \lambda_{x}=\int_{G} a_{0} d \lambda_{x} .
$$

From this fact we deduce the following 3 inequalities:

$$
\|b-a\|_{G}<\varepsilon, \quad\|b\|<1+\beta-\varepsilon, \quad\|b-a\|<\alpha-\beta-\varepsilon .
$$


If $\delta>0$ is small enough any function of the form $b+\psi$ where $\psi \in C(Y)$ with $\|\psi\|<\delta$ will satisfy the same 3 inequalities. The regularity of the measure $\mu$ and (*) then imply that

$$
\operatorname{Re}\left(\int(b+\psi) d \mu\right) \geqslant 0 \text { for } \psi \in C(Y),\|\psi\|<\delta .
$$

But $\mu$ annihilates $\bar{a}_{0}$ and hence $b$ so that $\mu$ has to be the zero measure, a contradiction.

Pick $a_{1} \in A_{0} \cap H$ and let $a_{2} \in A_{0}$ be an extension of $\left.a_{1}\right|_{G}-a_{0}$ with $\left\|a_{2}\right\|<\varepsilon$. Then $a^{\prime}=a_{1}-a_{2}$ has the required properties.

Proof of Theorem 2. Let $\left.h_{0} \in A_{0}\right|_{K \times F}$ be the function $h_{0}(z, x)$ $=\sum_{i=1}^{n} z_{i} b_{i}(x)$ for $(z, x) \in K \times F$ where $\left\{b_{1}, \ldots, b_{n}\right\}$ is the basis we picked out for $L$. Then from the definition of $K$ it follows that $\left\|h_{0}\right\|_{K \times F}=1$. It follows from the proof of Lemma 5 that there is a function $h \in A_{0}$ such that $\|h\|=1$ and $\left.h\right|_{K \times F}=h_{0}$. Let $c_{1}, \ldots, c_{n} \in B$ be the functions defining $h$, i.e., $h(z, x)=\sum_{i=1}^{n} z_{i} c_{i}(x)$ for all $(z, x) \in K \times X$. Now define a linear operator $u$ from $L$ into $B$ by

$$
u\left(\sum_{i=1}^{n} s_{i} b_{i}\right)=\sum_{i=1}^{n} s_{i} c_{i}
$$

where $s_{1}, \ldots, s_{n}$ are arbitrary complex numbers. It is clear that $u$ is an extension operator, i.e., that $\left.u\left(b_{0}\right)\right|_{F}=b_{0}$ for all $b_{0} \in L$. Let us find the norm of $u$. To this end let $b_{0} \in L$ with $\left\|b_{0}\right\|_{F}=1$. Then we can find an element $\left(z_{1}^{\prime}, \ldots, z_{n}^{\prime}\right)$ in $K$ such that $b_{0}(x)=\sum_{i=1}^{n} z_{i}^{\prime} b_{i}(x)$ for all $x \in F$ and hence $b_{0}(x)=h_{0}\left(z^{\prime}, x\right)$ for all $x \in F$. From the definition of $u$ we see that $u\left(b_{0}\right)(x)$ $=h\left(z^{\prime}, x\right)$ for all $x \in X$. Finally since $\|h\|=1$ we see that $\left\|u\left(b_{0}\right)\right\| \leqslant 1$ and it follows that $u$ has norm 1 . This concludes the proof of Theorem 2 .

To prove the main theorem we need a couple of propositions which show that for a given linear extension from a finite dimensional subspace of $C(X)$ there are linear extensions from larger finite dimensional subspaces which are close to the given one.

Proposition 6. Let $L \subseteq M$ be finite dimensional subspaces of $\left.B\right|_{F}$ and let $u$ be a linear extension operator of $L$ into $B$ of norm 1 . Then for each $\varepsilon>0$ there is a linear extension operator $v$ from $M$ into $B$ such that $\|u(a)+v(b)\|<1+\varepsilon$ if $a \in L, b \in M$ with $\|a+b\|_{F} \leqslant 1$ and $\|u(a)\| \leqslant 1$.

Proof. Let $\left\{b_{1}, \ldots, b_{k}\right\}$ be a basis for $L$ and let $\left\{b_{1}, \ldots, b_{k}, b_{k+1}, \ldots, b_{n}\right\}$ be a basis for $M$. Let $K$ be the set of points $z=\left(z_{1}, \ldots, z_{k+n}\right) \in C^{k+n}$ for which

$$
\left\|\sum_{i=1}^{k} z_{i} b_{i}\right\|_{F} \leqslant 1 \text { and }\left\|\sum_{i=1}^{k} z_{i} b_{i}+\sum_{j=1}^{n} z_{k+j} b_{j}\right\|_{F} \leqslant 1 .
$$

Since $\left\{b_{1}, \ldots, b_{n}\right\}$ is a linearly independent subset the set $K$ is a compact subset of $C^{k+n}$. We let $A$ be defined as in the proof of Theorem 2 relative to this new set $K$, but $A_{0}$ is now slightly different: 


$$
\begin{aligned}
& A_{0}=\left\{g \in C(K \times X): \exists c_{1}, \ldots, c_{n} \in B\right. \text { such that } \\
& \left.\qquad g(z, x)=\sum_{j=1}^{n} z_{k+j} c_{j}(x) \text { for all }(z, x) \in K \times X\right\}
\end{aligned}
$$

so that $A_{0}$ does not depend on the first $k$ coordinates of the points in $K$. As in the proof of Theorem 2 the set $K \times F$ is an $M$-set for $A$ and if $\mu \in A_{0}^{\perp}$ $\cap M\left(\partial_{A}\right)$ then $\left.\mu\right|_{K \times F} \in A_{0}^{\perp}$.

Let $h$ be the function in $A$ given by $h(z, x)=\sum_{i=1}^{k} z_{i} u\left(b_{i}\right)(x)$ for all $(z, x) \in K \times X$ and $\left.k_{0} \in A_{0}\right|_{K \times F}$ the function $k_{0}(z, x)=\sum_{j=1}^{n} z_{k+j} b_{j}(x)$ for $(z, x) \in K \times F$. If $h_{0}=\left.h\right|_{K \times F}$ then we have the following set up:

The set $K \times F$ is an $M$-set for $A$, and $A_{0}$ is a closed subspace of $A$ such that $\left.\mu\right|_{K \times F} \in A_{0}^{\perp}$ for all $\mu \in A_{0}^{\perp} \cap M\left(\partial_{A}\right)$. Further we have a function $h \in A$ and a function $\left.k_{0} \in A_{0}\right|_{K \times F}$ such that $\|h\| \leqslant 1$, and such that $\left\|h_{0}+k_{0}\right\|_{K \times F}$ $\leqslant 1$ where $h_{0}=\left.h\right|_{K \times F}$. The aim is to find an extension $k \in A_{0}$ of $k_{0}$ such that $\|h+k\|<1+\varepsilon$. To do so we put

$$
V=\left\{\phi \in C(K \times X):\left\|\left.\phi\right|_{K \times F}-k_{0}\right\|_{K \times F}<\varepsilon / 2,\|\phi+h\|<1+\varepsilon / 2\right\} .
$$

If $V \cap A_{0}=\varnothing$ then there is a nonzero measure $\mu \in A_{0}^{\perp}$ such that

$$
\operatorname{Re}\left(\int \phi d \mu\right)>0 \text { for all } \phi \in V \text {. }
$$

We define $\bar{k}_{0}$ in the same way as $\bar{a}_{0}$ was defined in the proof of Lemma 5 . As in that proof we find a $\delta>0$ such that

$$
\left\|h+\bar{k}_{0}+\psi\right\|<1+\varepsilon / 2 \text { for all } \psi \in C(K \times X) \text { with }\|\psi\|<\delta .
$$

Then, the regularity of the measure $\mu$ and (**) imply that

$$
\operatorname{Re}\left(\int\left(\bar{k}_{0}+\psi\right) d \mu\right) \geqslant 0 \text { for all } \psi \in C(K \times X),\|\psi\|<\delta .
$$

Since $\mu$ annihilates $\bar{k}_{0}$ this leads to a contradiction. Hence $V \cap A_{0} \neq \varnothing$. If $k_{1} \in V \cap A_{0}$ we let $k_{2} \in A_{0}$ be an extension of $k_{0}-\left.k_{1}\right|_{K \times F}$ with $\left\|k_{1}\right\|$ $<\varepsilon / 2$. Then we put $k=k_{1}+k_{2}$.

The extension $v$ from $M$ into $B$ is now defined as follows: If $c_{1}, \ldots, c_{n}$ are the functions in $B$ such that $k(z, x)=\sum_{j=1}^{n} z_{k+j} c_{j}(x)$ for all $(z, x) \in K \times X$ then we put $v\left(\sum_{j=1}^{n} s_{j} b_{j}\right)=\sum_{j=1}^{n} s_{j} c_{j}$ where $s_{1}, \ldots, s_{n}$ are arbitrary complex numbers. Then $v$ is a linear extension operator from $M$ into $B$ with the required properties.

Proposition 7. Let $L \subseteq M \subseteq N$ be finite dimensional subspaces of $\left.B\right|_{F}$ and suppose there is a linear operator $S$ of norm 1 from $N$ into $M$ such that $\|S-I\|_{L}<\varepsilon$. Let $u$ be a linear extension operator of norm 1 from $M$ into $B$. Then there is a linear extension operator $w$ of norm 1 from $N$ into $B$ such that $\|u-w\|_{L}<3 \varepsilon$.

Proof. By Proposition 6 there is a linear extension operator $v$ from $N$ into $B$ such that $\|u(a)+v(b)\|<1+\varepsilon$ whenever $a \in M, b \in N$ are such that $\|u(a)\| \leqslant 1$ and $\|a+b\|_{F} \leqslant 1$. We put $w^{\prime}(b)=u(S b)+v(b-S b)$. Then $\left\|w^{\prime}\right\|$ 
$<1+\varepsilon$. Using Lemma 5 with $A$ in place of $A_{0}$ we find another extension operator $w$ of norm 1 with $\left\|w-w^{\prime}\right\|<\varepsilon$.

We are now in a position to prove the main theorem. A separable Banach space $H$ is said to have the metric approximation property if there exists a sequence $\left\{T_{n}\right\}$ of operators of norm 1 with finite dimensional ranges from $H$ into $H$ such that $T_{n} x$ converges to $x$ for each $x \in H$. In this case we can find an increasing sequence $\left\{L_{n}\right\}$ of finite dimensional subspaces of $H$ with union dense in $H$, and a subsequence $\left\{S_{n}\right\}$ of $\left\{T_{n}\right\}$ such that $S_{n}$ maps $H$ into $L_{n}$ and such that $\left\|S_{n}-I\right\|_{L_{n-1}} \leqslant 2^{-n}$. Using Proposition 7 and proceeding as in [8] and [10] we obtain

THeOREM 8. Let $X$ be a compact Hausdorff space and $B$ a closed pointseparating subspace of $C(X)$ containing the constant functions. Further let $F$ be an $M$-set for $B$. Then if $H$ is a closed separable subspace of $\left.B\right|_{F}$ which possesses the metric approximation property there is a linear extension operator of norm 1 from $H$ into $B$.

If $\left.B\right|_{F}$ has the metric approximation property then $\left.B\right|_{F}$ is necessarily separable (and hence in particular $F$ is metrizable) Davie's construction [8, Theorem 1.1] shows that if $F$ is an $M$-set for a subspace $B$ of $C(X)$ and if $F$ is metric then $F$ can be embedded into a compact metric space $T$, and we can find a closed subspace $H$ of $C(T)$ with the metric approximation property such that $\left.H\right|_{F}=\left.B\right|_{F}$ and such that $F$ is an $M$-set for $H$. Thus, if in this case there exists a linear extension operator of norm 1 from $\left.B\right|_{F}$ into $H$ then $\left.B\right|_{F}$ must have the metric approximation property.

We now turn to the second problem of finding a linear inverse to the canonical map of $B$ onto $B / J$ where $J$ is a closed subspace of $B$ which is an $M$-ideal in $B$. As we wrote earlier there is an $M$-set $F$ for $B$ such that $J=\left\{b \in B:\left.b\right|_{F} \equiv 0\right\}$ [9]. If $F$ is an $M$-set then any function in the restriction space $\left.B\right|_{F}$ has a normpreserving extension to a function in $B$. This shows that $B / J$ is isometrically isomorphic to $\left.B\right|_{F}$. An application of Theorem 8 thus yields

THEOREM 9. Let $X$ be a compact Hausdorff space and $B$ a closed pointseparating subspace of $C(X)$ containing the constant functions. Let $J$ be a closed subspace of $B$ which is an $M$-ideal in $B$ and suppose $B / J$ has the metric approximation property. Then there is a linear inverse of norm 1 to the canonical map of $B$ onto $B / J$.

\section{REFERENCES}

1. E. M. Alfsen, Compact convex sets and boundary integrals, Springer-Verlag, Berlin, 1971.

2. E. M. Alfsen and E. Effros, Structure in real Banach spaces. I, II, Ann. of Math. (2) 96 (1972), 98-173. MR 50 \#5432.

3. E. M. Alfsen and B. Hirsberg, On dominated extensions in linear subspaces of $C_{C}(X)$, Pacific J. Math. 36 (1971), 567-584. MR 44 \#784.

4. T. B. Andersen, Linear extensions, projections and split faces, Univ. of Aarhus, Math. Inst., Preprint Series, no. 52, 1972/73.

5. T. Ando, Closed range theorems for convex sets and linear liftings, Pacific J. Math. 44 (1973), 393-410. MR 48 \#6888.

6. L. Asimow, Decomposable compact convex sets and peak sets for function spaces, Proc. Amer. Math. Soc. 25 (1970), 75-79. MR 41 \# 4244. 
7. E. Briem and M. Rao, Normpreserving extensions in subspaces of $C(X)$, Pacific. J. Math. 42 (1972), 581-588. MR 47 \#5572.

8. A. M. Davie, Linear extension operators for spaces and algebras of functions, Amer. J. Math. 94 (1972), 156-172. MR 45 \#9141.

9. B. Hirsberg, M-ideals in function spaces and algebras, Israel J. Math. 12 (1972), 133-146. MR 47 \# 4000.

10. E. Michael and A. Pełczyński, A linear extension theorem, Illinois J. Math. 11 (1967), 563-579. MR 36 \#671.

11. M. Rao, On simultaneous extensions, Invent. Math. 13 (1971), 284-294. MR 45 \#2458.

Department of Mathematics, Science Institute, Raunvisindastofnun Haskolans, UniVERSITY OF ICELAND, DUNHAGa 3, REYKJAVIK, ICELAND 